\title{
Serum miR-300 as a diagnostic and prognostic biomarker in osteosarcoma
}

\author{
JIAN-DONG LIU ${ }^{1,2}$, QUN XIN ${ }^{3}$, CHUN-SHENG TAO ${ }^{2}$, PEI-FENG SUN ${ }^{2}$, \\ $\mathrm{PENG} \mathrm{XU}^{2}$, BING WU ${ }^{2}$, LIANG QU ${ }^{1,2}$ and SHU-ZHONG $\mathrm{LI}^{1}$ \\ ${ }^{1}$ Department of Orthopedics, Affiliated Hospital of Qingdao University, Qingdao, Shandong 266000; \\ Departments of ${ }^{2}$ Orthopedics and ${ }^{3}$ General Surgery, 401 Hospital of PLA, Qingdao, Shandong 266071, P.R. China
}

Received June 9, 2015; Accepted August 26, 2016

DOI: $10.3892 / \mathrm{ol} .2016 .5214$

\begin{abstract}
In order to determine whether microRNA (miR)-300 is a diagnostic and prognostic biomarker in osteosarcoma, the miR-300 levels in serum of 114 osteosarcoma patients and 114 healthy controls were compared, followed by serum analysis of the differences between the pre-operative and post-operative sera of these osteosarcoma patients. It was observed that the concentration levels of miR-300 in the serum of osteosarcoma patients was significantly higher than those in the serum of healthy controls $(\mathrm{P}<0.01)$. Furthermore, the concentration levels of miR-300 in the post-operative serum were significantly reduced when compared with the pre-operative serum levels $(\mathrm{P}<0.001)$. High miR-300 levels in serum correlated significantly with clinical stage, distant metastasis and poor survival of osteosarcoma patients. Notably, serum miR-300 was an independent prognostic marker for osteosarcoma. In conclusion, our results suggested that serum miR-300 may be a potential and useful noninvasive biomarker for the early detection of osteosarcoma.
\end{abstract}

\section{Introduction}

Human osteosarcoma, mainly arising from osteoid tissue and producing immature bones, is the most common primary malignancy of the bone in adolescents (1-3), with an incidence rate of 4-5 cases/million worldwide (4). Despite current therapeutic strategies combining adjuvant chemotherapy, surgery and occasionally radiotherapy, the 5-year survival rate and

Correspondence to: Dr Liang Qu or Professor Shu-Zhong Li, Department of Orthopedics, Affiliated Hospital of Qingdao University, 16 Jiangsu Road, Qingdao, Shandong 266000, P.R. China E-mail: 318760661@qq.com

E-mail: qylsz8328@163.com

Abbreviations: miRNAs, microRNAs; RT-qPCR, reverse transcription-quantitative polymerase chain reaction; ROC, receiver operating characteristic; AUC, area under the curve

Key words: serum miR-300, diagnostic, prognosis, osteosarcoma prognosis of osteosarcoma patients remain poor, since there is a significant proportion of osteosarcoma patients with a high risk of local relapse or distant metastasis following curative resection of the primary tumor and intensive chemotherapy $(5,6)$. The 5-year survival rate of these patients is only $50-60 \%$, and nearly $40 \%$ of osteosarcoma patients succumb to lung metastases $(7,8)$. Therefore, it is urgently required to develop novel and efficient alternative strategies for the diagnosis of osteosarcoma patients at an early stage and for improved prognosis by selecting more efficient therapeutic approaches.

MicroRNAs (miRNAs or miRs) are non-coding RNAs of 18-25 nucleotides in length with highly conserved sequences across different species in plants, animals and DNA viruses $(9,10)$, which have been demonstrated to play a major role in the regulation of virtually all cellular processes and gene expression at the post-transcriptional level by binding to the 3'-untranslated region of their target messenger RNAs $(11,12)$. Recently, several studies have shown that aberrant miRNA expression is associated with the genesis and homeostasis of multiple types of cancer (13-19). Particularly in osteosarcoma, miR-21 was significantly overexpressed in osteosarcoma, and promoted invasion and migration in osteosarcoma cells (13). Subsequent studies also demonstrated that miR-100 (14), miR-204 (15), miR-144 (16), miR-195 (17), miR-26b (18) and miR-195 (19) were linked to the carcinogenesis, progression and prognosis of osteosarcoma. Furthermore, miRNAs have also been detected in human serum in remarkably stable forms (20), which makes serum miRNA patterns possible to be non-invasive cancer biomarkers with high sensitivity and specificity (21-23). As expected, numerous publications have reported that serum miRNAs exhibited different levels between cancer patients and healthy controls (24-26) and could served as stable blood-based biomarkers in various cancers $(27,28)$, which further highlighted the potential of circulating miRNAs as non-invasive diagnostic and prognostic biomarkers for cancerous diseases. Regarding osteosarcoma, recent studies revealed that the decreased expression of miR-195 in serum may be a novel biomarker for screening osteosarcoma and may predict poor prognosis (29). The muscle-specific miRNAs miR-133b and miR-206 were also demonstrated to be downregulated in the serum of osteosarcoma patients (30). Notably, serum miR-133b and miR-206 were all independent prognostic biomarkers for overall and progression-free 
survival of osteosarcoma patients (30). Serum miR-21 also was demonstrated to be a good candidate for a therapeutic target and a potential biomarker for the prediction of chemotherapeutic sensitivity and prognosis in patients with osteosarcoma (31).

A previous study revealed that miR-300 was increased in osteosarcoma tissues and cell lines compared with paired adjacent non-cancerous bone and osteoblastic cells (32). In addition, miR-300 was observed to promote proliferation and invasion of osteosarcoma cells (32). However, there are still no studies on the expression profile, diagnostic or prognostic significance of serum miR-300 in osteosarcoma patients.

In the current study, the role of miR-300 in osteosarcoma was systematically investigated by a two-phase study. In the first phase, quantitative analyses of miR-300 in a subset of serum samples from osteosarcoma patients and healthy control subjects were performed to determine the feasibility of its detection in the circulation. In the second phase, the clinical significance of miR-300 as a potential biomarker for diagnosis and prognosis of osteosarcoma patients was evaluated.

\section{Materials and methods}

Patients and specimens. Tissue and serum-based specimen collection and studies were approved by the Research Ethics Committee of the 401 Hospital of PLA (Qingdao, China). All patients provided written consent and indicated willingness to donate their blood and tissue samples for research. A total of 114 patients were enrolled in the present study, of whom, 97 patients received curative resection and 17 patients received palliative resection at the 401 Hospital of PLA from January 2004 to December 2009. None of the patients enrolled received radiotherapy or chemotherapy prior to the operation. All tumors were clinically and histologically diagnosed as osteosarcoma. Inclusion criteria for all cases included: i) Unambiguous histology and absence of mixed tumor types; and ii) absence of any treatment prior to surgery. The clinicopathological characteristics of the patients are presented in Table I. Patients were followed up subsequent to surgical treatment until March 2015, with a median follow-up time of 83 months (range, 12-131 months). During the follow-up period, 54 patients (47.4\%) succumbed to the disease.

RNA extraction and reverse transcription-quantitative polymerase chain reaction ( $R T-q P C R)$. For miRNA quantification, total miRNA was extracted from the sera of osteosarcoma patients and healthy controls using miRNeasy Mini kit (Qiagen, Inc., Valencia, CA, USA), according to the manufacturer's protocol. Synthetic cel-miR-39 (catalogue no. 219610; Qiagen, Inc.) was added as a spike-in control miRNA into each sample to normalize the sample-to-sample variation in the RNA isolation step and to detect the purification efficiency. TaqMan MicroRNA Reverse Transcription kit (Applied Biosystems; Thermo Fisher Scientific, Inc., Waltham, MA, USA) was used to detect and quantify the miRNA expression. Primer sequences used for PCR were as follows: Forward, 5'-TATACAAGGGCAGACTCTCTCT-3' and reverse, 5'-GTGCAGGTTCCGAGGT-3' for miR-300; forward, 5'-CTCGCTTCGGCAGCACATATACT-3' and reverse, 5'-ACGCTTCACGAATTTGCGTGTC-3' for U6. PCR was performed under the following conditions: 30 cycles of $95^{\circ} \mathrm{C}$ for $30 \mathrm{sec}, 57^{\circ} \mathrm{C}$ for $30 \mathrm{sec}$ and $72^{\circ} \mathrm{C}$ for $1 \mathrm{~min}$. Data were analyzed with ABI 7500 software v.2.0.1 (Applied Biosystems; Thermo Fisher Scientific, Inc.), with automatic quantification cycle $(\mathrm{Cq})$ settings for adapting baseline and threshold for $\mathrm{Cq}$ determination (32). Each sample was examined in triplicate, and the quantity of the PCR products was normalized to U6 (Applied Biosystems; Thermo Fisher Scientific, Inc.).

Statistical analyses. Statistical analysis was performed using SPSS statistical software version 21.0 (IBM SPSS, Armonk, NY, USA). Mann-Whitney U analyses of variance were used to evaluate statistical differences in serum miRNA expression between unpaired groups. The correlation of miR-300 expression between osteosarcoma tissues and serum was determined by Spearman's correlation analysis. The Wilcoxon signed-rank test was used to compare miR-300 expression in paired serum samples obtained prior to surgical tumor resection and 7 days after surgical tumor resection. Receiver operating characteristic (ROC) analysis was performed to determine the diagnostic performance of miR-300 expression levels in distinguishing patients with osteosarcoma from healthy control subjects. Sensitivity against $100 \%$ minus specificity was plotted for each cutoff threshold, and the area under the curve (AUC) values that reflected the probability of correctly identifying osteosarcoma patients from control subjects were computed. The optimal cutoff thresholds for diagnosis were obtained by the Youden's index. By using the optimal cutoff value, the sensitivity, specificity, and positive and negative predictive values were calculated. Survival curves were estimated using the Kaplan-Meier method, and distributions were evaluated by the long-rank test. Cox proportional hazard models of factors associated with survival were used to calculate the hazard ratios (HRs) and to identify factors that affect survival. The differences in characteristics between two groups were examined by the $\chi^{2}$ test and the Fisher's exact test. All P-values were determined from two-sided tests, and $\mathrm{P}<0.05$ was considered to indicate a statistically significant difference.

\section{Results}

Relative expression of serum miR-300 in osteosarcoma patients. The level of serum miR-300 in osteosarcoma patients was investigated. It was observed that the relative expression of serum miR-300 in 114 osteosarcoma patients was significantly higher than that in healthy controls $(\mathrm{P}=0.007$; Fig. 1A). Furthermore, the expression of serum miR-300 was significantly associated with the expression of tissue miR-300 in osteosarcoma patients (Spearman's correlation: $\mathrm{r}=0.25, \mathrm{P}=0.0068$; Fig. 1B).

\section{Diagnostic value of serum miR-300 expression in osteosarcoma patients. ROC curve analysis illustrated that serum miR-300 expression was a potential biomarker for discriminating osteo- sarcoma patients from healthy controls, with an AUC of 0.8847 (Fig. 2A). Using a cutoff value of 2.63, the sensitivity, specificity, and positive and negative predictive values were 84.2, 88.6, 89.7 and $84.9 \%$, respectively, to identify a patient with osteo- sarcoma.}

Alterations of serum miR-300 levels in patients with osteosarcoma. The serum levels of miR-300 significantly decreased following surgery in the same subset of osteosarcoma patients 
Table I. Association of miR-300 expression with clinicopathological features of osteosarcoma.

miR-300 expression

Clinicopathological features

No. of cases

$\operatorname{High}(\mathrm{n})$

Low (n)

P-value

Age (years)
$<55$
$\geq 55$

Gender

$\begin{array}{rrr}114 & 68 & 46 \\ 64 & 35 & 29 \\ 50 & 33 & 17\end{array}$

Male

58

37

0.446

Female

56

31

21

Tumor size (cm)

0.252

29

17

$\begin{array}{llll}>8 & 62 & 40 & 22 \\ \leq 8 & 52 & 28 & 24\end{array}$

Clinical stage

\begin{tabular}{lcccc} 
IIA & 48 & 22 & 26 & 20 \\
IIB/III & 66 & 46 & 3 & $0.035^{\mathrm{a}}$ \\
Metastasis & & & 15 & 3 \\
Present & 18 & 53 & 43 \\
Absent & 96 & 53 \\
\hline
\end{tabular}

${ }^{\mathrm{a}} \mathrm{P} \leq 0.05 . \mathrm{miR}$, microRNA.

A

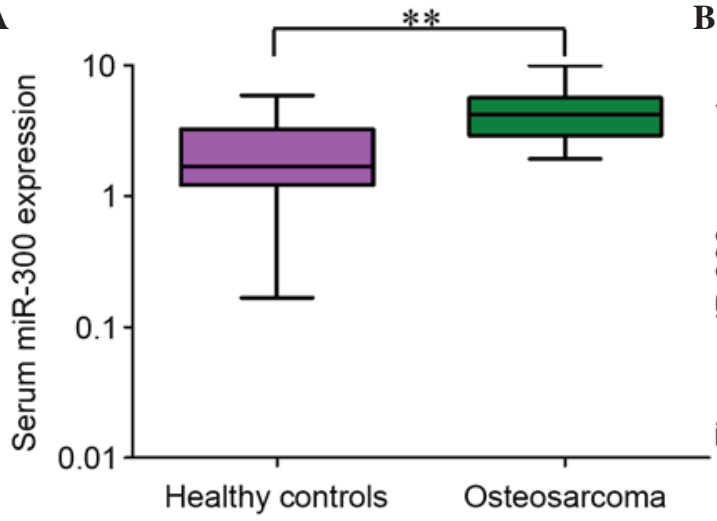

B

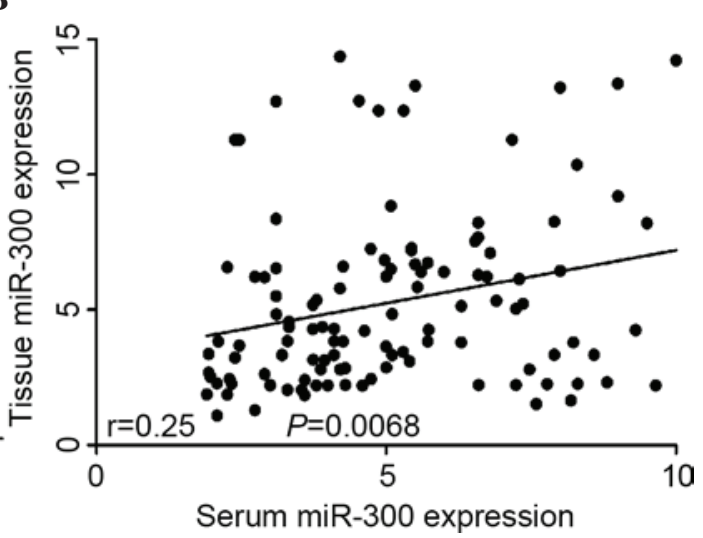

Figure 1. Expression of miR-300 in the serum of osteosarcoma patients. (A) Levels of serum miR-300 in normal controls and osteosarcoma patients. (B) Correlation of serum and tissue miR-300 levels in osteosarcoma patients. Mann-Whitney U test was used to analyze the significant differences. The correlation of miR-300 expression between osteosarcoma tissues and serum was determined by Spearman's correlation analysis. ${ }^{* *} \mathrm{P}<0.01$. miR, microRNA.

( $\mathrm{P}=0.009$; Fig. 2B). However, when data were analyzed based on potentially curative or palliative surgeries, serum miR-300 levels significantly decreased in patients with potentially curative surgeries ( $\mathrm{P}=0.0006$; Fig. 2C). Contrarily, no significant differences were observed in miR-300 levels prior or subsequent to surgery in patients with palliative resections (Fig. 2D).

Upregulation of serum miR-300 levels is associated with advanced clinicopathological characteristics of osteosarcoma patients. Next, it was investigated whether serum miR-300 expression correlated with clinicopathological characteristics of patients with osteosarcoma. As shown in Table I, serum miR-300 was significantly upregulated in osteosarcoma patients with advanced clinical stage $(\mathrm{P}=0.0120)$ and metastasis status $(\mathrm{P}=0.0350)$. However, there was no correlation of serum miR-300 expression with other clinical features such as age, gender or tumor size (all $\mathrm{P}>0.05$ ).

Upregulation of serum miR-300 levels is associated with poor overall survival and progression-free survival rates in osteosarcoma. The association between miR-300 expression and overall survival or progression-free survival was investigated using Kaplan-Meier analysis and log-rank test. Significant differences in overall survival and progression-free survival were detected between the high serum miR-300 expression group and the low serum miR-300 group (log-rank test: $\mathrm{P}=0.0498$ and $\mathrm{P}=0.0418$, respectively; Fig. $3 \mathrm{~A}$ and B). Patients with high serum miR-300 expression tended to have shorter 
A
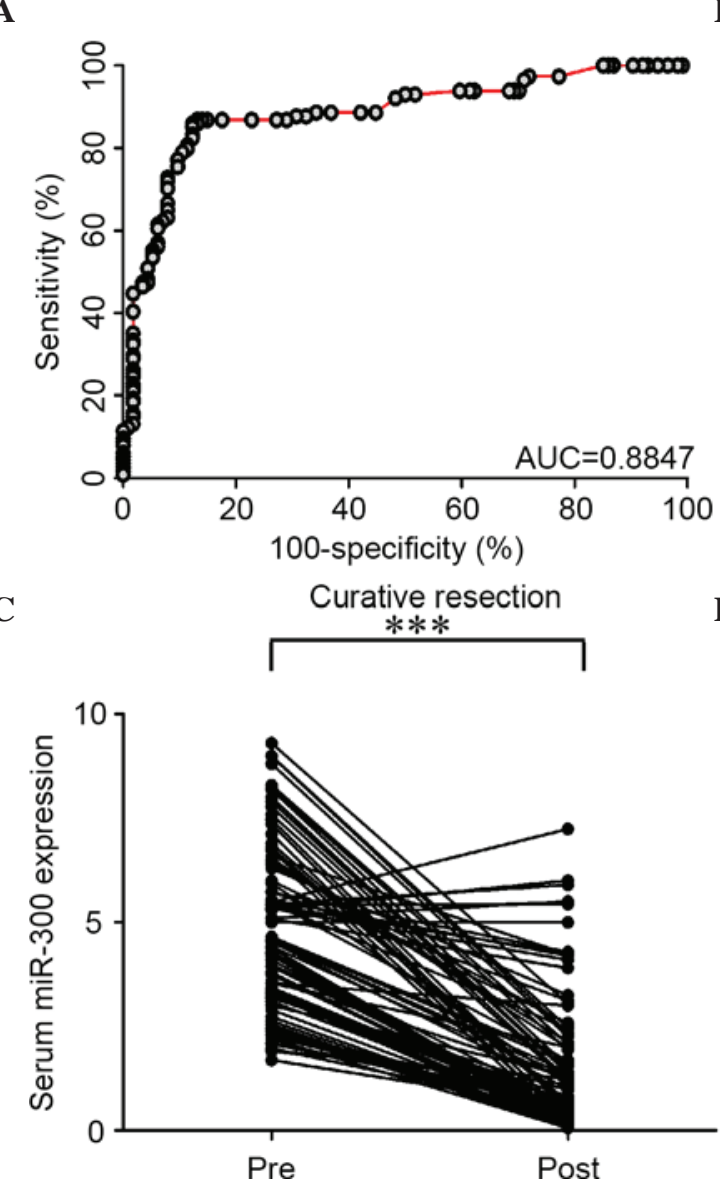
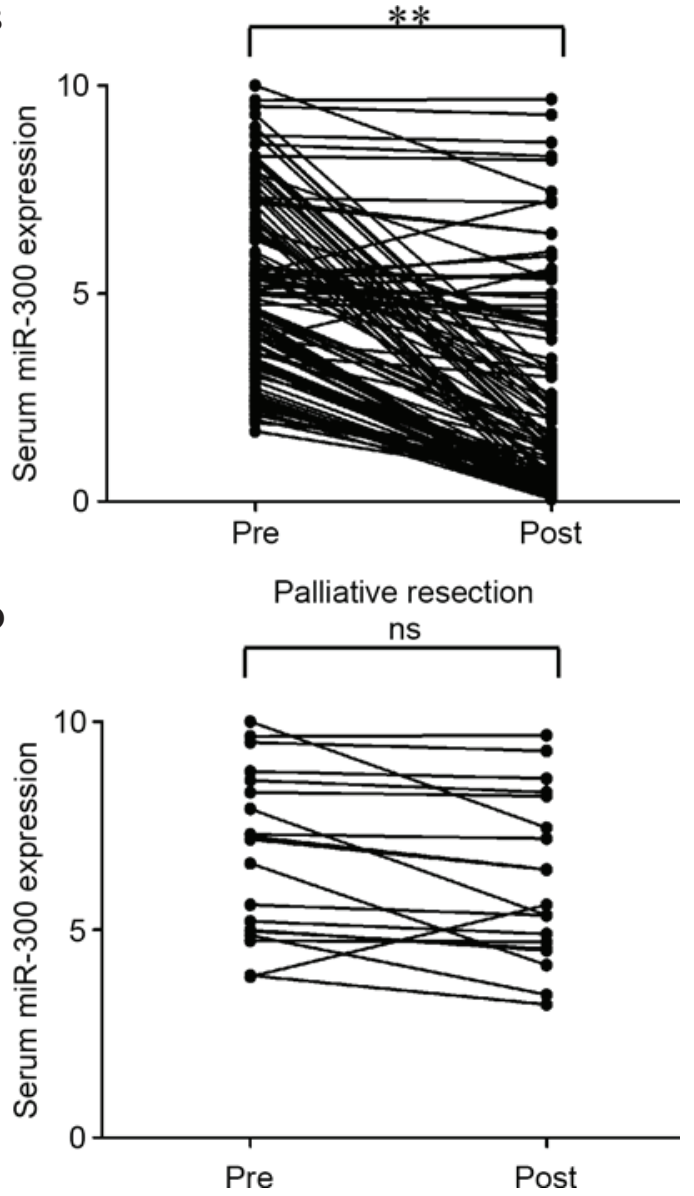

Figure 2. Serum miR-300 is a potential diagnostic biomarker for osteosarcoma patients. (A) Serum miR-300 yielded an AUC value of 0.8847 , with $84.2 \%$ sensitivity and $88.6 \%$ specificity in distinguishing osteosarcoma patients from normal control subjects. (B) Comparison of serum miR-300 levels from all osteosarcoma patients $(n=114)$. (C) Comparison of serum miR-300 levels in 97 osteosarcoma patients who underwent potentially curative surgeries. (D) Comparison of serum miR-300 in 17 osteosarcoma patients who underwent palliative resections. Two-tailed Student's t test was used to analyze the significant differences. ${ }^{* * *} \mathrm{P}<0.01,{ }^{* * *} \mathrm{P}<0.001 . \mathrm{miR}$, microRNA; AUC, area under the curve; ns, not significant.
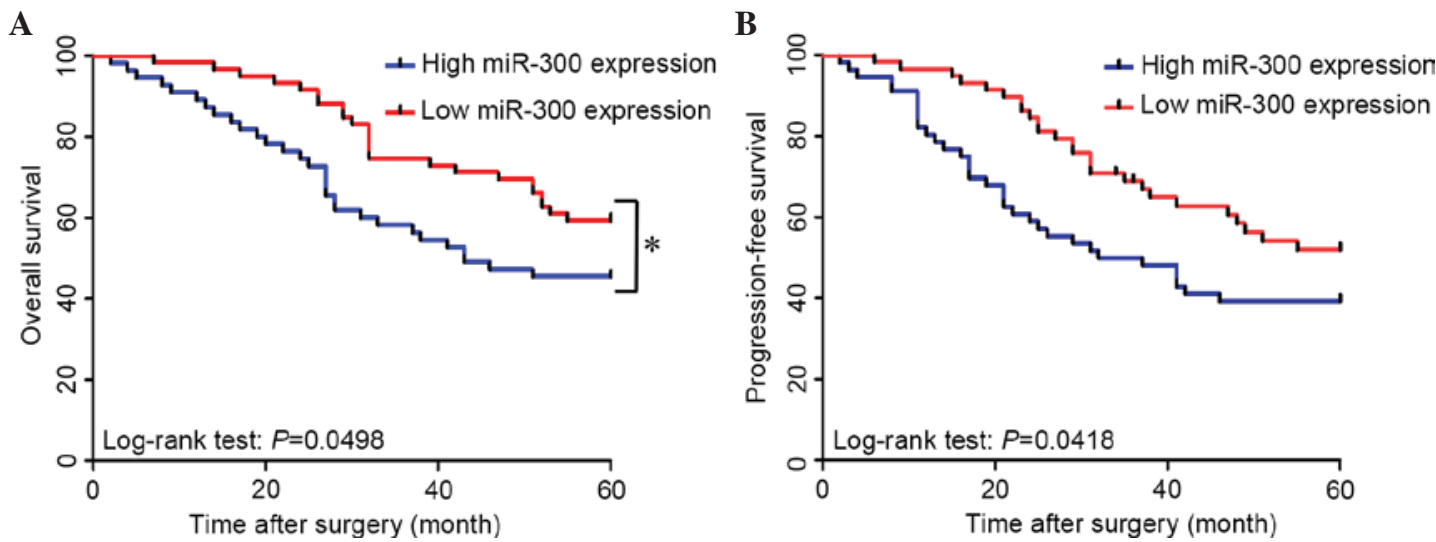

Figure 3. Association of serum miR-300 expression with overall and progression-free survival. (A and B) Kaplan-Meier graphs representing the probabilities of (A) overall survival and (B) progression-free survival in osteosarcoma patients according to their levels of serum miR-300. "P<0.05. Log-rank test was used to analyze the significant differences. miR, microRNA.

overall and progression-free survival times than patients with low serum miR-300 expression.

Upregulation of serum miR-300 levels is associated with poor prognosis in osteosarcoma patients. Univariate analysis identified clinical stage, metastasis status and high expression of serum miR-300 as poor prognostic factors for overall survival and progression-free survival (all $\mathrm{P}<0.05$; Table II), whereas neither age, gender or tumor size were significantly associated with overall survival or progression-free survival. To test whether the prognostic value of high serum miR-300 expression was independent of other risk factors for poor overall and progression-free survival, a multivariate analysis was performed using a Cox proportional hazard model. 
Table II. Univariate survival analysis of overall survival and disease-free survival in 114 patients with osteosarcoma.

\begin{tabular}{|c|c|c|c|c|c|c|}
\hline \multirow[b]{2}{*}{ Variables } & \multicolumn{3}{|c|}{ Overall survival } & \multicolumn{3}{|c|}{ Disease-free survival } \\
\hline & HR & $95 \% \mathrm{CI}$ & P-value & HR & $95 \% \mathrm{CI}$ & P-value \\
\hline miR-300 expression & 5.964 & $2.349-10.697$ & $0.009^{\mathrm{a}}$ & 5.112 & $2.136-9.482$ & $0.019^{\mathrm{a}}$ \\
\hline Clinical stage & 4.936 & $1.989-8.587$ & $0.011^{\mathrm{a}}$ & 3.216 & $2.102-6.964$ & $0.031^{\mathrm{a}}$ \\
\hline Metastasis status & 6.102 & $3.652-11.036$ & $0.006^{\mathrm{a}}$ & 5.563 & $2.645-8.254$ & $0.012^{\mathrm{a}}$ \\
\hline
\end{tabular}

${ }^{\mathrm{a}} \mathrm{P} \leq 0.05$. CI, confidence interval; HR, hazard ratio; miR, microRNA.

Table III. Multivariate survival analysis of overall survival and disease-free survival in 114 patients with osteosarcoma.

\begin{tabular}{|c|c|c|c|c|c|c|}
\hline \multirow[b]{2}{*}{ Variables } & \multicolumn{3}{|c|}{ Overall survival } & \multicolumn{3}{|c|}{ Disease-free survival } \\
\hline & HR & $95 \% \mathrm{CI}$ & P-value & HR & $95 \% \mathrm{CI}$ & P-value \\
\hline miR-300 expression & 4.698 & $1.562-8.369$ & $0.014^{\mathrm{a}}$ & 4.406 & $1.268-6.986$ & $0.013^{\mathrm{a}}$ \\
\hline Clinical stage & 3.869 & $1.269-6.858$ & $0.021^{\mathrm{a}}$ & 3.597 & $1.369-7.639$ & $0.029^{\mathrm{a}}$ \\
\hline Metastasis status & 3.165 & $1.197-5.459$ & $0.036^{\mathrm{a}}$ & 3.664 & $1.569-4.987$ & $0.027^{\mathrm{a}}$ \\
\hline
\end{tabular}

${ }^{\mathrm{a}} \mathrm{P} \leq 0.05$. CI, confidence interval; HR, hazard ratio; miR, microRNA.

Multivariate analyses including age, gender, tumor size, clinical stage, metastasis status and serum miR-300 expression demonstrated that high serum miR-300 expression was an independent predictor for poor overall and progression-free survival in osteosarcoma patients $[\mathrm{HR}=4.698,95 \%$ confidence interval $(\mathrm{CI})=1.562-8.369, \mathrm{P}=0.0140$ and $\mathrm{HR}=4.406,95 \%$ $\mathrm{CI}=1.268-6.986, \mathrm{P}=0.0130$, respectively; Table III). Significant results were also obtained for advanced clinical stage and metastasis status, whereas all other parameters were not significant independent prognostic markers for overall survival or progression-free survival.

\section{Discussion}

In the current study, the serum expression levels of miR-300 were detected in osteosarcoma patients and healthy controls, and their upregulation in the peripheral blood of osteosarcoma patients was confirmed. Furthermore, the diagnostic value of serum miR-300 in osteosarcoma was also evaluated by two steps. Initially, it was observed that the relative expression of serum miR-300 in osteosarcoma patients was significantly higher than that in healthy controls, and that the expression of serum miR-300 correlated significantly with the expression of tissue miR-300. The diagnostic value of serum miR-300 in the early detection of osetosarcoma was evaluated by yield AUC curves. It was observed that serum miR-300 could efficiently differentiate osteosarcoma patients from healthy controls (AUC $=0.8847$ ). Taken together, these results confirmed that the extraction of RNA and identification of miR-300 in the serum of individuals diagnosed with osteosarcoma is feasible, and offered the first description that miR-300 could be an effective diagnostic biomarker with high sensitivity and specificity for osteosarcoma.
Another important finding of our study is that serum miR-300 expression also serves as a prognostic biomarker for osteosarcoma. Our results are consistent with those from previous studies that demonstrated that miR-300 could promote cell proliferation and invasion in osteosarcoma cells (32). The present study identified the prognostic role of serum miR-300 in osteosarcoma by three steps. Initially, it was observed that the expression of serum miR-300 was significantly correlated with the expression of tissue miR-300. Then, it was noticed that serum miR-300 was also significantly correlated with progression-free and overall survival of osteosarcoma patients by Kaplan-Meier and univariate analyses. Finally, it was identified that serum miR-300 was an independent prognostic biomarker for patients with osteosarcoma by multivariate analysis. Our findings that high levels of serum miR-300 indicate a poor prognosis in patients with osteosarcoma are also an important step forward in the further identification of a non-invasive biomarker for osteosarcoma. To the best of our knowledge, the present study represents the first demonstration that serum miR-300 may not only be a diagnostic biomarker for osteosarcoma but may also aid to predict metastases or tumor recurrence with high accuracy.

The aberrant expression of miR-300 is a frequent event in multiple types of solid cancer, suggesting its important roles in carcinogenesis and cancer progression (32-34). Similar to other miRNAs, the expression pattern and functions of miR-300 may be different in various types of cancer, in a tissue-specific manner, dependent on the cellular context $(32,33)$. For example, Yu et al reported that miR-300 was downregulated in head and neck squamous cell carcinoma cells and in breast cancer cells. Downregulation of miR-300 was required for the initiation and maintenance of the transforming growth factor- $\beta$-induced epithelial-mesenchymal transition (EMT) (33). Thus, miR-300 
could inhibit cell invasion and metastasis by negatively regulating EMT in head and neck squamous cell carcinoma. By contrast, the expression of miR-300 was upregulated in glioma tissues and glioma stem-like cells, and overexpression of miR-300 could promote the self-renewal and cell proliferation of glioma stem-like cells (34). Similarly, the expression of miR-300 was also reported to be elevated in osteosarcoma tissues and cell lines compared with that in adjacent non-tumor bone tissues and osteoblastic cells (32). Notably, the overexpression of miR-300 could promote cell proliferation and invasion in osteosarcoma cells (32). In the present study, elevated serum miR-300 expression was identified in osteosarcoma patients, and its significant association with adverse clinicopathological features was confirmed, further suggesting the oncogenic role of miRNAs in osteosarcoma. These findings were consistent with those from a previous study, which reported that miR-300 could promote cell proliferation and cell invasion in vitro for the first time (32).

Although our current study indicates that serum miR-300 may be a promising screening tool for osteosarcoma, we acknowledge two potential limitations of using miR-300 as a single biomarker for the early detection of osteosarcoma. First, circulating expression of miR-300 has been described in numerous solid cancers besides osteosarcoma, including glioma and head and neck squamous cell carcinoma $(33,34)$. As a consequence, it may be challenging to differentiate whether circulating miR-300 expression is specifically associated with osteosarcoma itself or is a common phenomenon that manifests during the progression of any cancer as a result of perturbations in the host immune response $(35,36)$. Second, although it is highly unlikely to have a substantial impact, the use of serum miR-300 expression levels as a diagnostic and prognostic biomarker must be validated in diverse ethnic populations, since the clinical specimens analyzed in our study were solely from patients of Chinese origin.

In conclusion, the present study demonstrated that serum miR-300 was an effective diagnostic biomarker in osteosarcoma patients. Besides, it was also demonstrated that serum miR-300 levels were more frequently elevated in osteosarcoma patients with adverse clinical stage and presence of distant metastasis than osteosarcoma patients without adverse clinical stage and presence of distant metastasis. Multivariate survival analyses demonstrated that serum miR-300 was an independent prognostic factor for both progression-free and overall survival in osteosarcoma patients. Our results provide compelling evidence for the potential usefulness of serum miR-300 as a non-invasive screening tool and effective prognostic tool in patients with osteosarcoma.

\section{References}

1. Mirabello L, Troisi RJ and Savage SA: Osteosarcoma incidence and survival rates from 1973 to 2004: Data from the surveillance, epidemiology, and end results program. Cancer 115: 1531-1543, 2009.

2. Teicher BA: Searching for molecular targets in sarcoma. Biochem Pharmacol 84: 1-10, 2012.

3. Ottaviani $\mathrm{G}$ and Jaffe N: The epidemiology of osteosarcoma. Cancer Treat Res 152: 3-13, 2009.

4. KagerL,ZoubekA,PötschgerU,KastnerU,FlegeS,Kempf-BielackB, Branscheid D, Kotz R, Salzer-Kuntschik M, Winkelmann W, et al: Primary metastatic osteosarcoma: Presentation and outcome of patients treated on neoadjuvant cooperative osteosarcoma study group protocols. J Clin Oncol 21: 2011-2018, 2003.
5. Bacci G, Briccoli A, Rocca M, Ferrari S, Donati D, Longhi A, Bertoni F, Bacchini P, Giacomini S, Forni C, et al: Neoadjuvant chemotherapy for osteosarcoma of the extremities with metastases at presentation: Recent experience at the Rizzoli institute in 57 patients treated with cisplatin, doxorubicin, and a high dose of methotrexate and ifosfamide. Ann Oncol 14: 1126-1134, 2003.

6. Marina N, Gebhardt M, Teot L and Gorlick R: Biology and therapeutic advances for pediatric osteosarcoma. Oncologist 9: 422-441, 2004

7. Gorlick R: Current concepts on the molecular biology of osteosarcoma. Cancer Treat Res 152: 467-478, 2009

8. Bruland ØS, Bauer H, Alvegaard T and Smeland S: Treatment of osteosarcoma. The Scandinavian sarcoma group experience. Cancer Treat Res 152: 309-318, 2009.

9. Bentwich I, Avniel A, Karov Y, Aharonov R, Gilad S, Barad O, Barzilai A, Einat P, Einav U, Meiri E, et al: Identification of hundreds of conserved and nonconserved human microRNAs. Nat Genet 37: 766-770, 2005.

10. Bartel DP: MicroRNAs: Genomics, biogenesis, mechanism, and function. Cell 116: 281-297, 2004.

11. Ying $\mathrm{HC}, \mathrm{Xu} \mathrm{HY}, \mathrm{Lv} \mathrm{J}$, Ying TS and Yang Q: MicroRNA signatures of platinum-resistance in ovarian cancer. Eur J Gynaecol Oncol 36: 16-20, 2015.

12. Lim LP, Lau NC, Garrett-Engele P, Grimson A, Schelter JM, Castle J, Bartel DP, Linsley PS and Johnson JM: Microarray analysis shows that some microRNAs downregulate large numbers of target mRNAs. Nature 433: 769-773, 2005.

13. Ziyan W, Shuhua Y, Xiufang W and Xiaoyun L: MicroRNA-21 is involved in osteosarcoma cell invasion and migration. Med Oncol 28: 1469-1474, 2011.

14. Bi Y, Jing Y and Cao Y: Overexpression of miR-100 inhibits growth of osteosarcoma through FGFR3. Tumour Biol 36: 8405-8411, 2015.

15. Shi Y, Huang J, Zhou J, Liu Y, Fu X, Li Y, Yin G and Wen J: MicroRNA-204 inhibits proliferation, migration, invasion, and epithelial-mesenchymal transition in osteosarcoma cells via targeting Sirtuin 1. Oncol Rep 34: 399-406, 2015.

16. Wang W, Zhou X and Wei M: MicroRNA-144 suppresses osteosarcoma growth and metastasis by targeting ROCK 1 and ROCK2. Oncotarget 6: 10297-10308, 2015.

17. Han K, Chen X, Bian N, Ma B, Yang T, Cai C, Fan Q, Zhou Y and Zhao TB: MicroRNA profiling identifies MiR-195 suppresses osteosarcoma cell metastasis by targeting CCND1. Oncotarget 6: 8875-8889, 2015

18. Du JY, Wang LF, Wang Q and Yu LD: miR-26b inhibits proliferation, migration, invasion and apoptosis induction via the downregulation of 6-phosphofructo-2-kinase/fructose-2,6-bisphosphatase-3 driven glycolysis in osteosarcoma cells. Oncol Rep 33: 1890-1898, 2015.

19. Mao JH, Zhou RP, Peng AF, Liu ZL, Huang SH, Long XH and Shu Y: microRNA-195 suppresses osteosarcoma cell invasion and migration in vitro by targeting FASN. Oncol Lett 4: 1125-1129, 2012.

20. Calin GA and Croce CM: MicroRNA signatures in human cancers. Nat Rev Cancer 6: 857-866, 2006.

21. Kosaka N, Iguchi H and Ochiya T: Circulating microRNA in body fluid: A new potential biomarker for cancer diagnosis and prognosis. Cancer Sci 101: 2087-2092, 2010.

22. Chen X, Ba Y, Ma L, Cai X, Yin Y, Wang K, Guo J, Zhang Y, Chen J, Guo X, et al: Characterization of microRNAs in serum: A novel class of biomarkers for diagnosis of cancer and other diseases. Cell Res 18: 997-1006, 2008.

23. Lawrie CH, Gal S, Dunlop HM, Pushkaran B, Liggins AP, Pulford K, Banham AH, Pezzella F, Boultwood J, Wainscoat JS, et al: Detection of elevated levels of tumour-associated microRNAs in serum of patients with diffuse large B-cell lymphoma. Br J Haematol 141: 672-675, 2008.

24. Ng EK, Chong WW, Jin H, Lam EK, Shin VY, Yu J, Poon TC, Ng SS and Sung JJ: Differential expression of microRNAs in plasma of patients with colorectal cancer: A potential marker for colorectal cancer screening. Gut 58: 1375-1381, 2009.

25. Kong X, Du Y, Wang G, Gao J, Gong Y, Li L, Zhang Z, Zhu J, Jing Q, Qin Y and Li Z: Detection of differentially expressed microRNAs in serum of pancreatic ductal adenocarcinoma patients: miR-196a could be a potential marker for poor prognosis. Dig Dis Sci 56: 602-609, 2011.

26. Tsujiura M, Ichikawa D, Komatsu S, Shiozaki A, Takeshita H, Kosuga T, Konishi H, Morimura R, Deguchi K, Fujiwara H, et al: Circulating microRNAs in plasma of patients with gastric cancers. Br J Cancer 102: 1174-1179, 2010. 
27. Schwarzenbach H, Hoon DS and Pantel K: Cell-free nucleic acids as biomarkers in cancer patients. Nat Rev Cancer 11: 426-437, 2011.

28. Heneghan HM, Miller N, Lowery AJ, Sweeney KJ, Newell J and Kerin MJ: Circulating microRNAs as novel minimally invasive biomarkers for breast cancer. Ann Surg 251: 499-505, 2010.

29. Cai H, Zhao H, Tang J and Wu H: Serum miR-195 is a diagnostic and prognostic marker for osteosarcoma. J Surg Res 194: 505-510, 2015.

30. Zhang C, Yao C, Li H, Wang G and He X: Serum levels of microRNA-133b and microRNA-206 expression predict prognosis in patients with osteosarcoma. Int J Clin Exp Pathol 7: 4194-4203, 2014.

31. Yuan J, Chen L, Chen X, Sun W and Zhou X: Identification of serum microRNA-21 as a biomarker for chemosensitivity and prognosis in human osteosarcoma. J Int Med Res 40 2090-2097, 2012.

32. Xue Z, Zhao J, Niu L, An G, Guo Y and Ni L: Up-regulation of miR-300 promotes proliferation and invasion of osteosarcoma by targeting BRD7. PloS One 10: e0127682, 2015.
33. Yu J, Xie F, Bao X, Chen W and Xu Q: miR-300 inhibits epithelial to mesenchymal transition and metastasis by targeting twist in human epithelial cancer. Mol Cancer 13: 121, 2014.

34. Zhang D, Yang G, Chen X, Li C, Wang L, Liu Y, Han D, Liu H, Hou X, Zhang W, et al: mir-300 promotes self-renewal and inhibits the differentiation of glioma stem-like cells. J Mol Neurosci 53: 637-644, 2014

35. Xiao J, Luo X, Lin H, Zhang Y, Lu Y, Wang N, Zhang Y, Yang B and Wang Z: MicroRNA miR-133 represses HERG K+ channel expression contributing to QT prolongation in diabetic hearts. J Biol Chem 282: 12363-12367, 2007.

36. Toiyama Y, Takahashi M, Hur K, Nagasaka T, Tanaka K, Inoue Y, Kusunoki M, Boland CR and Goel A: Serum miR-21 as a diagnostic and prognostic biomarker in colorectal cancer. J Natl Cancer Inst 105: 849-859, 2013. 